\title{
Aussagekräftige Einkommensdaten dank transparenter Ärzteschaft
}

\author{
Jürg Schlup \\ Dr. med., Präsident der FMH
}

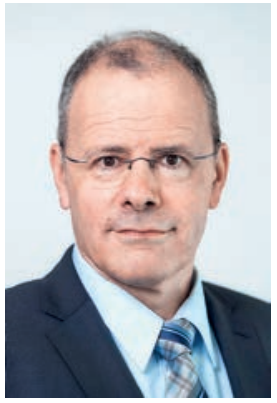

Obwohl Ärzteeinkommen in Medien und Politik regelmässig Aufmerksamkeit finden, werden die dazu vorhandenen Datengrundlagen oft ignoriert [1]. So hielt beispielsweise die Tatsache, dass tausende Ärzte seit 2016 detaillierte Einkommensdaten an das Bundesamt für Statistik (BFS) lieferten, das Bundesamt für Gesundheit (BAG) nicht davon ab, eine eigene Studie zum Thema anzukündigen [2]. Während die für Ende 2017 zugesagte Studie des BAG bis heute aussteht, wurden die Ergebnisse des BFS im April 2018 publiziert [3]. Auch wenn die MAS-Untersuchung des BFS keinerlei mediale oder politische Resonanz erhielt - und sogar in der Antwort auf eine parlamentarische Anfrage unerwähnt blieb [4] -, verdient sie grosse Beachtung. Denn weil knapp 7000 Ärztinnen und Ärzte umfangreiche Daten lieferten, erlaubt sie detaillierte Aussagen über den Aufwand und Ertrag von Arztpraxen - und über den durch die Prämien finanzierten Anteil: Gemäss BFS erzielten als Einzelunternehmen organisierte Arztpraxen im Jahr 2015 einen Umsatz von durchschnittlich 545000 Franken aus ihrer Berufstätigkeit, davon 508000 Franken aus ihrer Praxistätigkeit [3]. Da der Gesamtaufwand - also Kosten für Personal, Material, Räume etc. $-72 \%$ des Umsatzes ausmacht, beträgt das durchschnittliche Betriebsergebnis einer Einzelpraxis 155000 Franken [3].

Knapp 7000 Ärzte und Ärztinnen lieferten dem BFS umfangreiche Finanzdaten zu Umsatz, Aufwand und Betriebsergebnis

Berücksichtigt man, dass 89\% des Umsatzes aus Praxistätigkeit aus Geldern der obligatorischen Krankenpflegeversicherung generiert werden, bleiben im Ergebnis jährlich durchschnittlich 138000 Franken aus Prämiengeldern $(=155000 \times 0,89)$.

Um die Verlässlichkeit der MAS-Resultate zu prüfen, hat die FMH deren Daten mit ärzteeigenem Datenmaterial aus der RoKo-Studie verglichen. Unsere auf Seite 1480 nachlesbare Analyse zeigt: Der Bund und die FMH kommen zu vergleichbaren Resultaten [5]. Insofern dürften die MAS-Zahlen des BFS die Einkommen der freipraktizierenden Ärzteschaft realistisch abbilden.

Die Zahlen der MAS-Studie sind aus zwei Gründen ein grosser Fortschritt gegenüber den früher von der FMH gemeinsam mit dem Büro Bass publizierten Zahlen. Erstens ist eindeutig, wer untersucht wurde, nämlich ausschliesslich Arztpraxen und ambulante Zentren. Dies vermeidet die Verzerrungen der früheren Studien, die alle selbständig abrechnenden Ärzte betrachteten. Dies schloss auch Chefärzte und leitende Ärzte von

Nach den Zahlen des BFS bleibt der durchschnittlichen Einzelpraxis ein Brutto-Ergebnis von 138000 Franken aus Prämiengeldern

Spitälern ein, obwohl diese den Grossteil ihrer Einkommen nicht aus dem ambulanten Tarif oder Prämiengeldern, sondern aus ihrer Spitalanstellung erzielen. Hingegen stellen Assistenz- und Oberärzte ein Drittel aller Ärzte und rechnen nicht selbständig ab. Somit bleibt in allen Diskussionen um Ärzteeinkommen dasjenige Drittel der berufstätigen Ärzteschaft mit dem tiefsten Einkommen systematisch unberücksichtigt.

Der zweite Vorteil der hier präsentierten Daten ist, dass sie klar zeigen, wie die Einkommen generiert werden: Der Prämienzahler kann genau erkennen, wie viel Geld ein Praxisinhaber aus der solidarischen Prämienfinanzierung erhält. Bei den früher in der FMH-BassStudie verwendeten AHV-pflichtigen Einkommen blieb hingegen offen, inwieweit diese aus Prämiengeldern, aus Praxistätigkeit oder überhaupt aus ärztlichen Tätigkeiten resultierten.

Valide Daten zu den Einkommen der praxisambulanten Ärzteschaft helfen allerdings nur dann weiter, wenn man die verfügbaren Informationen auch nutzt - und nicht das selektive Skandalisieren von Einzelfällen und das Politisieren mit fragwürdigen Zahlen zu Millioneneinkommen [6] fortsetzt. Die FMH stellt sich gerne einer solchen fairen und faktenbasierten Diskussion! 